\title{
HISTORIA
}

\section{BARBEROS Y SANGRADORES FLEBOTOMIANOS EN GRANADA: NORMA Y SOCIEDAD EN LOS SIGLOS XVII Y XVIII}

\author{
MANUEL AMEZCUA \\ Laboratorio de Antropología Cultural, Universidad de Granada \\ Dirección para correspondencia: \\ Manuel Amezcua. Apartado de correos nº 73418080 Granada.
}

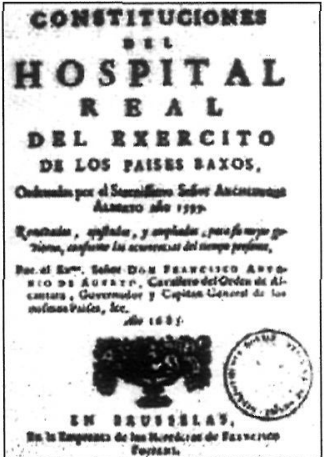

\section{RESUMEN}

Los barberos sangradores y los sangradores flebotomianos fueron quizá los profesionales más característicos de la práctica empírica de la medicina en la España Moderna. Su ejercicio estuvo asociado a la vigencia de una técnica delegada de la medicina, la sangría, que diera lugar a sesudos enfrentamientos entre partidarios y detractores de sus indicaciones y eficacia terapéutica. Los sangradores alcanzaron un cierto reconocimiento social, pero su práctica siempre estuvo condicionada por su posición ambigua entre los profesionales titulados (médicos y cirujanos latinos) y otros empíricos sin formación cuyos métodos traspasaban a menudo los límites de la superstición.

En el Archivo Histórico de la ciudad de Granada, se conservan algunos títulos de sangradores flebotomianos, expedidos entre 1666 y 1720, cuyo análisis se realiza en este trabajo con el objeto de ofrecer una visión aproximada del tipo de profesional que era el sangrador, así como algunas características en particular del sangrador granadino. Estos documentos se presentaron en el cabildo de la ciudad por sus titulares al tiempo de solicitar licencia para ejercer en ella, siendo en total siete los títulos que se conservan íntegros más otros tres de los que sólo se aportan algunos datos. En uno de los casos se inserta también el título de cirujano, lo que nos permite esclarecer las diferencias tanto formativas como funcionales respecto del sangrador.

$\mathrm{El}$ análisis de los documentos se realiza desde la perspectiva del contexto social donde fueron emanados, contraponiendo las cosideraciones legales del ejercicio de las distintas disciplinas auxiliares de la medicina (cirujanos, barberos, sangradores) con el nivel de socialización que alcanzaron, para lo que se manejan algunas fuentes literarias. 


\title{
BARBERS AND PHLEBOTOMISTS IN GRANADA: RULES AND SOCIETY IN THE XVII AND XVIII CENTURIES
}

\author{
SUMMARY
}

The barbers and phlebotomists were perhaps the most characteristic professionals of empirical practice in medicine in modern Spain. Their work was associated with a medical technique: 'Bleeding', which was the cause of dramatic battles between pro-bleeding and anti-bleeding groups which found themselves embroiled in bitter discussions about its therapeutic value. Bleeders reached some social standing at the time but their practise had always been questioned due to their ambiguous position between fully qualified professionals - doctors and latin surgeons - and those other empirical amateurs with no education other than experience, whose methods could somehow be compared to those of superstition or witchcraft. The skillful work of barber and bleeder was permanently under control of the 'Protomedicato,' an institution created by the Reyes Catolicos (The Catholic Kings) in order to govern and maintain the high quality of healthcare practised by medical doctors and surgeons. The institution was later extended to cover some other jobs loosely associated with medicine, such as enamelists, chemists, and herb specialists. Barbers and bleeders were allowed to work for rather a short period in Castille, where all the disciplineswere merged by the 'Protobarbeirato', while in Valencia their legal endorsement to work was common to barbers, surgeons and bleeders. They all had to sit for the same type of exam under latin surgeons.

In the Granada Town Hall Library some certificates of phlebotomian bleeders issued between 1666 and 1720 are preserved. Our present study concentrates on these documents with the main purpose of analysing the profession of bleeder at that time, and especially in Granada. There are 7 certificates in prime condition while in some other 3, only a few facts are legible. One of the documents shows the difference between a doctor's and a bleeder's function, not only at a theoretical level but at in practise as well. Our analysis is performed from a social perspective, considering the diverse aspects of the many core disciplines: surgeon, bleeder, barber..., as well as the other auxiliary medical practises prevalent at the time.

\section{INTRODUCCIÓN}

Los barberos sangradores y los sangradores flebotomianos fueron quizá los profesionales más característicos de la práctica empírica de la medicina en la España Moderna. Su ejercicio estuvo asociado a la vigencia de una técnica delegada de la medicina, la sangría, que diera lugar a sesudos enfrentamientos entre partidarios y detractores de sus indicaciones y eficacia terapéutica. Los sangradores alcanzaron un cierto reconocimiento social, pero su práctica siempre estuvo condicionada por su posición ambigua entre los profesionales titulados (médicos y cirujanos latinos) y otros empíricos sin formación cuyos métodos traspasaban a menudo los límites de la superstición.

El ejercicio de barberos y sangradores fue controlado tempr anamente por el Protomedicato, institución fundada por los Reyes Católicos con el objeto de regular el quehacer curador de médicos y cirujanos, pero cuyas competencias se extendieron a otros oficios con ellos relacionados como ensalmadores, boticarios, especieros o herbolarios. En el caso de los barberos y sangradores, esta facultad permaneció por poco tiempo en Castilla, donde fue absorbida por las competencias del Protobarbeirato, mientras que permaneció en Valencia por ser un mismo título el de barbero, cirujano y sangrador, siendo examinados por cirujanos latinos (Muñoz ME, 1751:61).

El Protobarbeirato, según pragmática del año 1500 , tenía que velar porque sólo ejercieran aquellos barberos que fueran examinados por los barberos mayores. Se refería a aquellos barberos que pusieran tienda para sajar, a quienes se autorizaba para sacar dientes y muelas, sangrar y poner ventosas y sanguijuelas, mientras que no se entrometían en el oficio de los que sólo ponían tienda de afeitar. En tiempos de Felipe II una nueva reglamentación prevenía que los protomédicos no se entrometieran en examinar a especieros, parteras, ensalmadores, drogueros, ni otras personas inhábiles por las muchas vejaciones a que les sometían Iborra P, 1987:28), con lo que el Protobarbeirato se consolidaba como entidad independiente. 


\section{Barberos y sangradores}

En el Archivo Histórico de la ciudad de Granada, se conservan algunos títulos de sangradores flebotomianos, expedidos entre 1666 y 1720, cuyo análisis nos puede dar una visión aproximada del tipo de profesional que era el sangrador, así como algunas características en particular del sangrador granadino. Estos documentos se presentaron en el cabildo de la ciudad por sus titulares al tiempo de solicitar licencia para ejercer en ella, siendo en total siete los títulos que se conservan íntegros más otros tres de los que sólo se aportan algunos datos. En uno de los casos se inserta también el título de cirujano, lo que nos permite esclarecer las diferencias tanto formativas como funcionales respecto del sangrador.

La mayoría de los sangradores que exhiben su título son naturales de la ciudad de Granada, siendo los menos de la provincia (Iznalloz, Alhendín) o de fuera de ella (Lucena). Sus edades se sitúan entre los 22 y 39 años, predominando los más jóvenes (fig. 1). Como curiosidad y en base a la descripción física de los interesados que contienen sus títulos, podemos decir que predominan los de mediana estatura, y como rasgo de identidad la presencia de cicatrices en los dedos de la mano izquierda, sin duda consecuencia de la torpeza en el manejo del escalpelo mientras se iniciaron en su arte.

Todos fueron examinados por el Protobarbeirato, por sangradores del rey que actuaban como alcaldes y examinadores, siendo la mayoría expedidos en la villa de Madrid y los menos en la propia ciudad de Granada (fig. 1). En el título de sangrador expedido a Juan Hervás (1685), que se transcribe al final de este capítulo, quedan reflejadas perfectamente las condiciones que debía reunir el aspirante para alcanzar la facultad de sangrar. Para ser recibido a examen era preciso haber practicado el arte de sangrador flebotomiano durante al menos cuatro años bajo la tutoría de un maestro examinado, circunstancia que había de acreditar la justicia local.

El examen constaba de una parte teórica y una práctica. En la primera el aspirante debía exponer sus conocimientos sobre la anatomía vascular y los procedimientos más rutinarios del arte: del conocimiento de las venas, cuáles y cuántas son y en qué lugares se reparten, y de los nombres de ellas y del modo de sangrar, sajar, hechar ventosas, sanguijuelas, sacar dientes y muelas, mientras que en la segunda, realizada en un hospital, debía demostrar su habilidad práctica en las técnicas de sangrar, sajar, echar ventosas, poner sanguijuelas y sacar dientes y muelas. De esta forma el título de sangrador flebotomiano le facultaba no sólo para estos menesteres, sino también para que pueda sangrar en las enfermedades de dolor de costado, erisipela, mal de garganta y caída no habiendo médico aprobado que las ordene'.

El título de sangrador tenía validez para todos los reinos y señoríos de su Majestad y llevaba implícito el juramento de su titular de ejercer su arte de balde para los pobres. Los derechos de examen se fijaban en media annata, que era el equivalente a la mitad de los emolumentos que podía producir el empleo en un año.

\section{Barberos y Cirujanos}

Aunque actuaba como profesional liberal, el barbero sangrador, junto a otros oficiales y dependientes, formaba parte de la plantilla de todo hospital importante. Es precisamente en el ámbito institucional donde se pone de manifiesto la dependencia funcional del médico. Así se expresan las obligaciones del barbero y cirujano del Hospital Real al tiempo de su fundación:

Ha de acudir el Cirujano, y Barbero todos los dias a visitar los enfermos a las mismas horas que el Médico los visitare, y quando en su oficio aya alguna dificultad, consultarla ha con el Médico, y no ha de poner sostituto en su lugar, sino servir siempre por su propia persona; y quando tuviere algdn legítimo impedimiento para no acudir a el Hospital, en tal caso sea obligado a embiar otro en su lugar, con licencia, y aprouación del Administrador, y no de otra manera y hasele de dar el salario que ha lleuado hasta aquí (Constituciones del Hospital Real... 1671: 15v).

Al tenor de este documento puede parecer confusa la diferenciación entre el, barbero sangrador y el cirujano, que sin duda gozaba de mayor consideración social que el primero. El cirujano romancista, a diferencia del latinista, formaba parte también del amplio espectro de empíricos que rodeaban a la práctica médica. Uno de los sangradores que solicitaron 
ejercer en Granada, Juan Francisco de Aguilar, se examinó en 1673 también para obtener el título de cirujano y algebrista. Lo hizo en el Protomedicato y hubo de acreditar previamente que había practicado dichas artes durante cinco años, siendo las materias de que fue examinado la anatomía del cuerpo vmano y en el conocimiento y curación de las heridas y llagas'.

Otro sangrador que también ejerció como cirujano fue el granadino Ambrosio Martínez (1687), cuyos títulos obtuvo con nueve años de diferencia. De hecho era frecuente que los barberos sangradores aspirasen a obtener el título de cirujanos, que sin duda gozaría en la práctica liberal de mejores emolumentos. Así parece deducirse del anuncio que insertaba en 1765 el Semanero Granadino:

Un mancebo de 18 años muy aplicado, $y$ honesto, que sabe afeitar muy bien, pretende acomodarse solo por la comida, dandole lugar, y tiempo para ir a la Academia a aprender la Cirugía, que solicita professar. En esta imprenta el Oficial dará razón (Gacetilla curiosa... 1765).

A pesar de su condición de empíricos, los barberos sangradores contaron con una profusión de tratados en los que estudiar su práctica, de los que Granjel identifica hasta cinco títulos impresos en el siglo XVII (Sánchez Granjel, 1978:194), entre ellos la Instrucción de los barberos flebotomianos de Alonso Muñoz (1621). Sin embargo la obra más completa y ambiciosa del género permaneció inexplicablemente inédita, se trata del Directorio de enfermeros (1652) de Simón López, un enfermero y barbero que ejerció en los hospitales de Valladolid y Salamanca cuya obra ha sido estudiada por Carreras Panchón (1973) y García Martínez (1994).

La consideración social alcanzada por los barberos sangradores, en cita de Granjel (1978:195), es puesta de manifiesto por Cervantes cuando a Maese Nicolás, barbero del innominado lugar de la Mancha, patria de Don Quijote, le hace compartir sus ocios con el cura y con el propio Alonso Quijano, dotado de una cultura tal que le permitirá colaborar con el cura en el expurgo de la biblioteca del ingenioso hidalgo. Aunque, todo hay que decirlo, son más las recreaciones burlescas sobre el personaje, pudiendo bastar como ejemplo este retrato que Tirso de Molina pone en boca de un personaje de sus comedias:
Ha estudiado cirugía; no hay hombre más afamado; agora imprime un tratado todo de flosomonía.

Suele andar en un machuelo, que en vez de caminar vuela; sin parar saca una muela; más almas tienen en el cielo que un Herodes y un Nerón; conócenle en cada casa: por donde quiera que pasa le llaman la Extrema Unción.

Sin duda resulta edificante la lectura del viejo documento que proponemos como anexo, el título de sangrador flebotomiano de Juan de Hervás, que ya comenté en parte en otra ocasión (Amezcua, 1994), pues tras él se adivinan las peripecias que un pintoresco profesional de los Montes Orientales de Granada, como tantos otros de la provincia, tuvo que atravesar hasta realizar ese viaje iniciático a la corte para traerse a cambio de unos pocos reales el pergamino que le autorizase a ejercer su profesión con una mediana dignidad.

\section{Notas}

1. Archivo Municipal de Granada. Solicitudes y expedientes de sangradores, barberos y sacamuelas. Leg. n! 57 (+193). Letra E.

\section{BIBLIOGRAFÍA}

Amezcua, M (1994). El perfil de un sangrador flebotomiano de Iznalloz en el siglo XVII. Bolet Inform (Gran). 1994; 36:27-28.

Carreras Panchón, A (1973). "Enfermeros y Barberos en el siglo XVII según el manuscrito de Simón López". Actas del IV Congreso Español de Historia de la Medicina. Granada, vol. III: 247-250. 
García Martínez, M.J., Valle Racero JI, García Martínez AC (1994). La edificante doctrina del perfecto enfermero, por Simón López. Index de Enfermería, 8-9: 66-70.

Iborra, P. (1987). Historia del Protomedicato en España (1477-1822). Valladolid: Universidad, Acta Histórico-Médica Vallisoletana XXIV. Muñoz, M.E. (1751). Recopilación de las leyes, pragmáticas reales, decretos y acuerdos del Real Protomedicato. Valencia: Impr. Vda. de Bordázar.
Sánchez Granjel, L. (1978). La medicina Española del siglo XVII. Salamanca: Universidad, pág.

Sin autor (1671). Constituciones del Hospital Real que en la ciudad de Granada fundaron los señores Reyes Católicos D. Fernando y Doña Isabel. Granada: Imprenta Real.

Sin autor (1765). Gazetilla curiosa, o semanero granadino, noticioso, y util para el bien común. 3 de junio, papel LXI.

\title{
ANEXO 1
}

\section{TITULO DE SANGRADOR FLEBOTOMIANO DE JUAN HERVAS Natural de Iznalloz. Año 1686}

\author{
Fuente: Archivo Municipal de Granada
}

Nos, Juan Rodriguez Aguado, Luis Muñoz Azero y Joseph Ximénez de Bonilla, Sangradores del Rey nuestro Señor y sus proto barberos generales, Alcaldes y examinadores mayores en todos sus reinos y señorios de todos los sangradores flebotomianos, hacemos saber a los que la presente vieran que ante nos apareció presente Juan de Hervás, natural de la villa de Iznalloz, diócesis de Granada, que es un hombre de mediana estatura, que tiene el brazo derecho encogido y dislocado por el codo y una cicatriz de herida en el mismo codo, y nos hizo relación diciendo que había practicado el arte de sangrador flebotomiano los cuatro años que su Majestad manda, como constaba de información que presentó hecha por autoridad de justicia, y atento a la prática él quería ser examinado en el arte y visto por nos su petición y la información la dimos por buena y le admitimos a examen y le examinamos en la teórica del arte acerca del conocimiento de las venas, cuáles y cuántas son y en qué lugares se reparten, y de los nombres de ellas y del modo de sangrar, sajar, hechar ventosas, sanguijuelas, sacar dientes y muelas, y en un hospital le vimos obrar de manos haciendo sangrías, a todo lo cual satisfizo y respondió bien y cumplidamente y por nos visto su habilidad y suficiencia y la buena cuenta y razón que en el examen dió le aprobamos y por la presente damos licencia y facultad cumplida a Juan de Hervás para que libremente sin pena ni calumnia alguna pueda usar y ejercer el arte de sangrador flebotominano en todos los casos y cosas a él tocantes y concernientes en todas las ciudades, villas y lugares de los reinos y señoríos de su Majestad, y para que pueda sangrar en las enfermedades de dolor de costado, erisipela, mal de garganta y caida, no habiendo médico aprobado que las ordene, por cuanto para ello le hicimos las preguntas necessarias y de qué venas conviene se hagan las sangrias en dichas enfermedades y del susodicho recibimos juramento de que bien y fielmente usará el dicho arte y a los pobres hará limosna en el llevar de su trabajo y prometió cumplirlo y hacerlo así. Por tanto de parte del Rey nuestro Señor exhortamos y requerimos a todos y a cualesquier juez y justicia que le dejen y consientan usar el dicho arte sin ponerle embargo ni impedimiento alguno ni consientan que sobre ello sea vejado ni molestado, antes le guarden y hagan guardar todas las honras, gracias, mros, franquezas y libertades que a semejantes maestros aprobados suelen y deben ser guardadas, haciéndole pagar qualesquier maravedis y otras cosas que por el uso de dicho su arte le fueran debidas y de ello le mandamos dar y dimos este título y licencia signado de Manuel López, escribano del Rey nuestro Señor y perpetuo de nuestros exámenes de que ha pagado el derecho de la media Anata el dicho Juan de Hervás, dada en la villa de Madrid a veintitrés de septiembre año de mil seiscientos ochenta y tres. Juan Rodríguez Aguado (rúbrica) y Luis Muñoz Azero (rúbrica) y Joseph Ximénez de Bonilla (rúbrica). 


\title{
Figura 1
}

\section{SANGRADORES QUE SOLICITARON LICENCIA PARA EJERCER EN LA CIUDAD DE GRANADA ENTRE 1666 Y 1720}

\author{
Fuente: Archivo Municipal de Granada
}

\begin{tabular}{|c|c|c|c|c|c|}
\hline Sangrador & Naturaleza & Edad & Expedido en & Fecha & Protobarberos examinadores \\
\hline $\begin{array}{l}\text { Diego Domínguez de } \\
\text { Arroyo }\end{array}$ & Granada & 22 & Madrid & 10.09.1666 & $\begin{array}{l}\text { Juan Rodríguez Abarza, } \\
\text { Juan Muñoz y Francisco de } \\
\text { Giberio Suaco }\end{array}$ \\
\hline Lorenzo de Arroyo & Granada & 25 & Madrid & 11.12.1669 & Idem \\
\hline Juan Francisco de Aguilar & Lucena & 22 & Madrid & 10.05 .1673 & $\begin{array}{l}\text { Juan Rodríguez Abarza, } \\
\text { Francisco de Giberio Suaco } \\
\text { y Juan Rodríguez Aguado }\end{array}$ \\
\hline Ambrosio Martínez & Granada & & Madrid & 30.04 .1673 & \\
\hline Juan de Hervás & Iznalloz & & Madrid & 23.09 .1683 & $\begin{array}{l}\text { Juan Rodríguez Aguado, } \\
\text { Luis Muñoz Azero y José } \\
\text { Ximénez de Bonilla }\end{array}$ \\
\hline Baltasar López del Arbol & Granada & 39 & Granada & 10.03 .1685 & Blas López \\
\hline Juan de Robles Caravaca & & & Granada & 13.03 .1685 & Idem \\
\hline Juan González de Meneses & Granada & & Granada & 14.03 .1685 & Idem \\
\hline Miguel Martínez & Granada & & Madrid & 24.10 .1686 & $\begin{array}{l}\text { Luis Muñoz Azero y José } \\
\text { Ximénez de Bonilla }\end{array}$ \\
\hline Lorenzo Martínez & Alhendín & & Madrid & 25.10 .1720 & $\begin{array}{l}\text { Juan Bautista Lexendre, } \\
\text { Ricardo Leprende y José } \\
\text { Fontana }\end{array}$ \\
\hline
\end{tabular}

